\title{
PENSIERI INTEMPESTIVI '17-'18 RIFLESSIONI SULLA RILETTURA ODIERNA DELLA PUBBLICISTICA DI M. GOR'KIJ
}

\author{
LJUDMILA CHAPOVALOVA
}

ABSTRACT. - Nel 1990 a Mosca è uscito un libro di M. Gor'kij dal titolo Nesvoevremennye mysli. Zametki o revoljucii i kul'ture (Pensieri intempestivi. Note su rivoluzione e cultura); si trattava di una raccolta di 58 articoli scritti e pubblicati dallo scrittore su Novaja Źizn' (Vita Nuova) dall'aprile del 1917 al luglio del 1918, quando il giornale fu chiuso per disposizione di V. Lenin.

Questo volume, arrivato al lettore russo solo settanta anni dopo, suscitò grande risonanza ed interesse.

In questo libro il Gor'kij "ben noto" si presenta in vesti assolutamente insolite, mostrando nuovi lati della sua personalità ricca ed altrettanto complessa.

Dopo la perestrojka, con la caduta della censura e la riconquista della libertà di parola, è stato possibile l'accesso agli archivi, una volta chiusi, e ad altri documenti, che permisero un nuovo approccio nello studio dello scrittore.

I Pensieri intempestivi riflettono la fase di maggior accentuazione delle contraddizioni dello scrittore, i suoi dubbi sul significato della rivoluzione russa, sul ruolo dell'intelligencija, sulla democrazia e sulla libertà.

Questi articoli sono la testimonianza inequivocabile della posizione di M. Gor'kij alla vigilia e nei primi mesi della rivoluzione d'Ottobre, posizione che si distacca decisamente da quella ufficialmente presentata e conosciuta nell'Unione Sovietica.

Non troveremo in Pensieri intempestivi quella presuntuosa convinzione ed univocità che caratterizzavano i suoi articoli del periodo precedente Zametki o meščanstve (Note sulla piccola borghesia)(1905), Razrušenie licnosti (Distruzione della personalità) (1909) e quelli degli anni 1930 (creati nella “prigionia di Stalin”), ma una tormentosa ricerca di significati e di risposte. Proprio in questo consistono i suoi pregi, la sua forza e vitalità, la sua modernità ed attualità.

La rilettura odierna del testo gor'koviano, ci permette non solo di verificare 
la vera posizione di M. Gor'kij nei confronti degli eventi rivoluzionari, ma ci ripropone questioni che rimangono attuali e di vitale importanza anche per le società contemporanee.

$* * *$

В 1990 году в Москве вышла книга М. Горького “Несвоевременные мысли. Заметки о революции и культуре”; это был сборник, состоящий из 58 статей, которые писатель публиковал на страницах газеты “Новая жизнь” с апреля 1917 по июль 1918, когда по распоряжению Ленина газета была закрыта как оппозиционный орган печати.

Эта книга дошла до читателей семьдесят лет спустя (в последующий советский период она ни разу не переиздавалась, о ней никогда и нигде не упоминалось, даже в академическом издании полного собрания сочинений М. Горького ) и вызвала огромный интерес. В этом сборнике “хорошо известный” Горький представал в совершенно необычном обличье, проявляя новые стороны своей противоречивой и сложной личности.

Перестройка с последующей отменой цензуры, свобода слова, открытие доступа к ранее закрытым архивам и документам, - все это позволяет и требует нового подхода к прочтению и изучению творческого наследия М. Горького.

Сложный творческий путь М. Горького писателя, публициста, выдающейся личности в социальной жизни России начала $\mathrm{XX}$ века проходит через надежды и горькие разочарования, через веру и отчаяние, через твердые убеждения и мучительные сомнения. "Несвоевременные мысли” отражают фазу наибольшего столкновения противоречий писателя, его напряженные поиски ответов на вопросы о значении русской революции, о роли интеллигенции, о демократии и о свободе. В то же время "Несвоевременные мысли" представляют собой сумму размышлений Горького об этих проблемах.

В этих статьях представлена совершенно определенная, недвусмысленная без чьей - либо интерпретации позиция писателя накануне и в первые месяцы Октябрьской революции, позиция, которая решительно отличается от той, что была официально представлена и широко известна в Советском Союзе.

В “Несвоевременных мыслях" мы не обнаружим ту высокомерную самоуверенную убежденность и однозначность, которые так характерны для горьковских статей предыдущего периода («Заметки о мещанстве» (1905), «Разрушение личности» (1909), и для работ 1930-х годов (написанных уже в сталинский период), - именно в этом заключается их ценность, сила и жизненность, их своевременность и современность.

Суть этих статей заключается в постановке самых жгучих и сложных, часто неразрешимых вопросов нашего существования, и в мучительных поисках ответов на них.

В 1917 году основные усилия М. Горького направлены на защиту культуры: «одной из первых задач момента должно быть возбуждение в народе - рядом с возбужденными в нем эмоциями политическими - эмоций этических и эстетических» $<\ldots>$ Можно и еще много сказать на тему о необходимости немедленной и упорной культурной работы в нашей стране. Мне кажется, что возглас «Отечество в опасно- 
сти!» не так страшен, как возглас: «Граждане! Культура в опасности!» - заявляет писатель. Не следует верить в то, что революция оздоровит и духовно обогатит Россию, подчеркивает М. Горький, - только после победы революции начнется процесс интеллектуального роста, который сам по себе очень медленный.

М. Горький обращается к представителям всех классов с призывом взяться за работу всем вместе и развивать все формы культуры; используя тот факт, что революция смела все препятствия на пути свободного творчества, следует показать и себе, и всему миру дар, талант и гений народа.

Совершенно неожиданными и непривычными предстают в этих статьях многочисленные резкие суждения о руководителях большевиков: “Ленин, Троцкий и сопутствующие им уже отравились гнилым ядом власти"; часто встречаются предупреждения: " Рабочий класс не может не понять, что Ленин на его шкуре, на его крови производит только некий опыт” или “ Ленин не всемогущий чародей, а хладнокровный фокусник, не жалеющий ни чести, ни жизни пролетариата.”

Современное прочтение горьковского текста позволяет не только узнать истинную позицию писателя в отношении революционных событий, но и с новой силой ставит перед нами вопросы, которые остаются важными и актуальными и для современного общества

M. Gor'kij divenne una figura di spicco a partire dagli anni '90 del XIX secolo e nel corso della sua vita rimase al centro dell'attenzione della società russa ed internazionale. ${ }^{1}$ Diverse erano e tuttora sono le valutazioni e le percezioni della sua attività letteraria, della sua posizione politica, ideologica e civile; fu senza dubbio un punto di riferimento e di attrazione per intellettuali, artisti, rivoluzionari di vario tipo.

E' noto che nel periodo sovietico intorno alla figura di M. Gor'kij fu creato un mito che lo presentava come primo e migliore scrittore sovietico, l'iniziatore della letteratura sovietica, il fondatore del realismo socialista, amico personale e fedele compagno di lotta di V. Lenin ecc. Il potere sovietico da una parte ricopriva M. Gor'kij di tutte le onorificenze, di privilegi e comodità, dall'altra parte sfruttava al massimo il suo nome e il prestigio della sua autorità per finalità propagan-

1. M. Gor'kij compì un viaggio in America, visitò Londra e Parigi, ma l'Italia occupò un posto particolare nella sua biografia letteraria e intellettuale: vi trascorse circa 15 anni: dall'ottobre del 1906 al dicembre 1913 (il suo soggiorno sull'isola di Capri) e dall'aprile 1924 al maggio 1933 (il soggiorno sorrentino frammezzato, verso la fine, da viaggi nell'URSS). A questo argomento è dedicata un'ampia letteratura tra cui un volume recente a cura di M. Talalay Uno scrittore 'amaro' nel paese 'dolce', OEBALUS, Capri, 2006. 
distiche. Questo riguardava anche lo studio della produzione artistica di M. Gor'kij: facevano parte dei programmi scolastici e delle università quelle opere i cui personaggi, problematiche, concezioni di vita dello scrittore, divenuto la "procellaria della rivoluzione", lo presentavano come combattente convinto della rivoluzione socialista; così M. Gor'kij risultò nella stretta cornice (anche se dorata) di una certa interpretazione ideologica.

Oggi, alla luce di tutto ciò che si è appreso nell'arco di un secolo, grazie alla conoscenza di documenti e fatti nuovi, la complessa e contraddittoria figura di Gor'kij ${ }^{2}$ è sottoposta ad un radicale ripensamento. In questo processo un ruolo importante è quello svolto dalla pubblicazione nel 1990 a Mosca d'un libro di M. Gor'kij dal titolo Nesvoevremennye mysli. Zametki o revoljucii i kul'ture (Pensieri intempestivi. Riflessioni sulla rivoluzione e la cultura), ${ }^{3}$ che settantacinque anni dopo, acquista un valore ancora maggiore, come si può constatare dalla sua lettura alla luce dell'esperienza storica.

L'uscita di questo volume, tenuto nascosto o passato sotto silenzio, per così lungo tempo (l'unica edizione russa che comprendeva 48 articoli della Novaja Źizn' risale al 1918 a Pietroburgo), suscitò grande risonanza e vivissimo interesse. Nell'introduzione all'edizione italiana dei Pensieri intempestivi $i^{4}$ firmata da German Ermolaev, troviamo una dettagliata descrizione del processo in cui il silenzio, le omissioni, le scelte mirate delle citazioni tolte dal loro contesto fecero sì che quest'opera pubblicistica di M. Gor'kij rimanesse sconosciuta nella sua integrità. ${ }^{5} \mathrm{Si}$ trattava di una raccolta di 58 articoli scritti e pubblicati dallo scrittore sul giornale social-democratico Novaja Źizn' (Vita Nuova) dall'aprile del

2. Evg. Zamjatin che ha potuto lasciare l'URSS nel 1931 grazie all'intervento di M. Gor'kij presso Stalin, diceva che nel suo benefattore coabitavano due persone in una: Aleksej Peškov, morto nel 1936, e Maksim Gor'kij, che è sopravvissuto; oggi si possono individuare più di due persone in quest'uomo la cui vita è tutta un libro, un "romanzo avvincente", come dice lo stesso Zamjatin.

3. M. Gor'kij. Nesvoevremennye mysli. Zametki o revoljucii i kul'ture. M., Sovetskij pisatel', 1990. e M. Gor'kij. Nesvoevremennye mysli. M., Sovremennik, 1991. In ital. Maksim Gorkij. Pensieri intempestivi '17-'18, Jaca Book, Milano, 1978.

4. Maksim Gor'kij. Pensieri intempestivi, 1917-1918, testi raccolti e annotati da German Ermolaev, prefazione di Boris Suvarin, trad. di Paolo Repetto, Edizioni Jaca Book, Milano, 1978.

5. Ibidem, pp. 26-29. 
1917 al luglio del 1918, quando il giornale fu chiuso, perchè organo d'opposizione, per disposizione di V. Lenin. ${ }^{6}$

Dalle pagine di questo libro il Gor'kij "ben noto" ai sovietici si presentava in vesti assolutamente insolite, mostrando nuovi lati della sua personalità ricca ed altrettanto complessa.

Durante la perestrojka, caratterizzata dal rifiuto dei miti e degli idoli sovietici, si sviluppa la stessa tendenza nei confronti di Gor'kij; negli anni successivi però l'immagine dello scrittore si è appianata, fatto al quale hanno contribuito la caduta della censura, ricerche in archivi prima inaccessibili e lo studio di nuove memorie e documenti non consultabili prima. Oggi possiamo parlare della ripresa degli studi dell'eredità gor'koviana in un contesto nuovo.

Il percorso di M. Gor'kij artista, pubblicista, personalità di spicco nella vita sociale della Russia dell'inizio del '900 è tormentato, segnato dalla speranza e da amare delusioni, in bilico tra la fede e la sfiducia, la ferma convinzione e rovinosi dubbi. I Pensieri intempestivi riflettono la fase di maggior accentuazione delle contraddizioni dello scrittore, le sue intense ricerche di risposte sul significato della rivoluzione russa, sul ruolo dell'intelligencija, sulla democrazia e sulla libertà. Nello stesso tempo i Pensieri intempestivi rappresentano la summa delle riflessioni di Gor'kij su questi problemi, e sono indispensabili alla comprensione delle sue idee.

Leggendo questi articoli, in cui emergono con tutta l'evidenza le contraddizioni della personalità di Gor'kij nelle sue riflessioni sulla rivoluzione, la cultura, il popolo e le realtà del periodo 1917-1918, il lettore percepisce in modo inequivocabile e trasparente la posizione di M. Gor'kij e le sue emozioni alla vigilia e nei primi mesi della rivoluzione d'Ottobre, posizione che si distacca decisamente da quella ufficialmente presentata e conosciuta nel periodo sovietico.

Non a caso come sottotitolo dei Pensieri intempestivi veniva messo Note sulla rivoluzione e cultura (Zametki o revoljucii i kul'ture). Il titolo esprime volutamente la posizione di M. Gor'kij che in quel contesto appariva inopportuna con la sua richiesta pressante di un'immediata soluzione di tanti problemi. Lo scrittore riteneva che il compito primario

6. Dopo la soppressione della Novaja Źizn' nel luglio del 1918, M. Gor'kij si dedica essenzialmente a salvaguardare l'intelligencija in pericolo e si occupa della sorte di innumerevoli perseguitati che vedono in lui un aiuto estremo. 
della rivoluzione sociale consistesse nella cura dello spirito e della morale degli uomini, e la soluzione non poteva che passare attraverso la cultura, l'educazione culturale.

Ricorderemo che da giovane M. Gor'kij fu attirato dalle idee del socialismo, e con ardore innato dichiarava che il socialismo avrebbe dovuto diventare la nuova religione del proletariato che salverà il mondo.

Si considerava socialista nel senso più ampio e meno dottrinario del termine, "eretico ovunque" come dice lui stesso, pronto a dare il suo appoggio morale e soprattutto il suo aiuto materiale ai diversi partiti socialisti. La politica nel senso meschino della parola gli era estranea: "Ho un disgusto organico per la politica, e sono un marxista pieno di dubbi", scriveva non senza ragione, allorché il marxismo "alla russa" divenne qualcosa di schematico e di elementare, sempre più lontano dal suo contenuto originario.

Sono questi e altri ancora le dichiarazioni, le idee e i punti di vista che, a partire dal 1909, portarono Gor'kij alle aspre polemiche e agli scontri continui con Lenin e i suoi collaboratori.

L'argomento più spesso discusso riguarda l'intelligencija. E' utile, a questo proposito, ricordare due testi pubblicistici di M. Gor'kij: $\mathrm{Za}$ metki o meščanstve (Note sulla piccola borghesia, 1905) e Razrušenie ličnost $\imath^{8}$ (Distruzione della personalità, 1909), dove per la prima volta fu affrontato il tema dell'intelligencija e della rivoluzione.

Nel primo articolo il concetto di meščianstvo ("piccolo borghese") è inteso come struttura e stato dell'anima, la cui caratteristica principale è il sentimento, sviluppato in modo deforme, della proprietà, l'intenso desiderio di quiete dentro e fuori di sé, un'oscura paura di fronte a tutto ciò che in qualche modo possa disturbare questa quiete. L'autore arriva alla conclusione che in fin dei conti questo non sia altro che "oscura paura davanti alla vita, davanti al popolo". Questo stato d'animo, secondo Gor'kij, appartiene all'intelligencija russa che teme la parola "democrazia".

Gor'kij come la maggior parte dell'intelligencija, accoglie con entusiasmo l'arrivo della rivoluzione, ma dice che la classe operaia deve dissociarsi, separarsi dagli intelligenty di stampo piccolo borghese.

7. L'articolo Zametki o meščanstve fu pubblicato nel giornale Novaja Żizn' nel novembre-dicembre del 1905 in pieno sviluppo degli eventi rivoluzionari a Mosca.

8. L'articolo "Razrušenie ličnosti" (si incontra nelle lettere di Gor'kij anche con un altro titolo Personalità e creatività) fu pubblicato per la prima volta nel volume Saggi di filosofia del collettivismo, raccolta I, ed. "Znanie”, Sbb, 1909. 
Conducendo alla logica conclusione il pensiero di Gor'kij sulla rivoluzione che "porta con sé non solo i giorni di lotta, ma pure giorni di giudizio", si arriva al finale minaccioso, per cui l'intelligencija è vista come zavorra per la nazione della quale bisogna liberarsi.

Il primo articolo scritto in modo duro, aspro persino provocatorio e strabiliante proprio per questi aspetti piacque a Lenin e suscitò l'indignazione nell'intelligencija liberale, rappresentata dalla voce di $\mathrm{N}$. Berdjaev che criticò le posizioni di Gor'kij sull'intelligencija accusandolo di "servilismo davanti al proletariato", di "teppismo culturale e politico", di mancanza di rispetto verso la cultura del passato, di profanazione della libertà in nome della "libertà".

Nel secondo articolo la distruzione della personalità di cui parla M. Gor'kij è riferita sempre alla distruzione dell'anima dell'intelligencija di stampo piccolo borghese. Sviluppando l'argomento centrale sottolinea il primato del collettivo, affermando che la singola personalità in contrapposizione al collettivo è condannata. Questa posizione di Gor'kij è legata anzitutto alle idee di A. Bogdanov - filosofo socialista, teorico della cultura proletaria (Proletkult).

A. Blok interpretò in modo diverso le ricerche di Gor'kij intervenendo con una relazione "Popolo e Intelligencija" Gor'kij sono due figure chiave dell'epoca, rappresentano due poli della vita e della cultura russa del Novecento, affrontano gli stessi problemi ma da posizioni diverse.

Risulta interessante l'evoluzione del pensiero di M. Gor'kij sull'intelligencija, sulla rivoluzione e sul popolo avvenuta nel periodo dall'inizio del '900 al 1917, quando cominciò a pubblicare le sue "note sulla rivoluzione e la cultura" ${ }^{11}$ che costituirono in seguito i Pensieri intempestivi.

Nel 1906 a Philadelphia intervenendo in un comizio M. Gor'kij parlava della Russia che si trovava alla soglia della rivoluzione che avrebbe portato libertà. Lo scrittore esprimeva la speranza che non fosse versato del sangue, ma "se verrà versato sangue la colpa cadrebbe sullo zar russo... Sarebbe sbagliato considerare la rivoluzione iniziata

9. N. Berdjaev. Revoljucija i kul'tura (Rivoluzione e cultura), Poljarnaja zvezda, $1905, \mathrm{n}^{\circ} 2,22$ dic.

10. Pubblicato con il titolo modificato: A. Blok. La Russia e l'Intelligencija, Zolotoe runo, $\mathrm{n}^{\mathrm{o}} 1 ; 1909$.

11. Il titolo di queste note rievoca l'articolo di N. Berdjaev Revolucija $i$ kul'tura con la quale Gor'kij polemizzava nel suo Razrušenie ličnosti. 
dal popolo come una semplice ribellione di plebe affamata provocata dalla disperazione. No, sarà un movimento di gente civile guidata dalla necessità cosciente di libertà, di vita e di lavoro per il bene del popolo."

Alla vigilia della rivoluzione russa del 1905-1907 M. Gor'kij in quanto oppositore del potere zarista si unì ai bolscevichi, ma già nel 1909 nascono i contrasti con V. Lenin e con la linea del partito che diventeranno sempre più profondi negli anni 1917-1918, di conseguenza la sua valutazione e il rapporto nei confronti della rivoluzione non furono univoci. Lo scrittore protestava con decisione contro il terrore, rosso o bianco che fosse, si tormentava per il destino della cultura russa che veniva distrutta. Guardava il socialismo attraverso il prisma della libertà e della democrazia, ma la realtà della guerra civile distruggeva questo mito.

Subito dopo la rivoluzione di Febbraio, accolta con grande entusiasmo, lo scrittore si dedicò completamente all'attività di pubblicista nel "risuscitato" giornale Novaja Žizn' che fu pensato dalla redazione e dallo stesso M. Gor'kij, come momento di consolidamento di tutte le forze sane della società senza distinzioni di appartenenza politica. Come compito principale proponeva un'ampia propaganda di valori spirituali e delle culture nazionali, il che prevedeva contatti con il governo borghese per l'organizzazione del lavoro nel campo della cultura e dell'istruzione del popolo. M. Gor'kij secondo la testimonianza di I. Gronskij, cercava all'inizio del 1917 di "unire tutte le correnti socialdemocratiche in un partito".

Già nel primo numero del giornale (18 aprile del 1917), M. Gor'kij scriveva: "Il vecchio regime era inetto, ma l'istinto di conservazione gli suggeriva non senza ragione che non c'è nemico più pericoloso del cervello umano: esso si sforzava dunque, in tutti i modi possibili, d'intralciare o di falsare la crescita delle forze intellettuali del paese." ${ }^{12}$ La prima guerra Mondiale, - continua -, ha rivelato con tutta evidenza "i risultati di questo lento processo di soffocamento dello spirito", però "Non si dovrebbe credere che la rivoluzione abbia spiritualmente guarito ed arricchito la Russia". ${ }^{13}$

Soltanto dopo la vittoria della rivoluzione inizierà "il processo di arricchimento intellettuale di un paese che è estremamente lento", avverte M. Gor'kij.

Lo scrittore si rivolge ai rappresentanti di "tutte le classi": "Dob-

12. Maksim Gor'kij. Pensieri intempestivi, 1917-1918, Ed. Jaca Book, Milano, 1978, p. 35 .

13. Ibidem. p. 37. 
biamo metterci all'opera tutti assieme affinché venga sviluppata ogni forma di cultura: la rivoluzione ha rimosso gli ostacoli sulla via della libera creazione: a noi ora mostrare al mondo, così come a noi stessi, le nostre doti, i nostri talenti e il nostro ingegno".

Come si vede in questo periodo e in quello immediatamente successivo alla rivoluzione d'Ottobre, il massimo impegno di M. Gor'kij è rivolto alla difesa della cultura: "Uno dei primi obiettivi del momento attuale dovrebbe essere quello di risvegliare nel popolo, accanto alle emozioni politiche già suscitate in esso, delle emozioni etiche ed estetiche", insiste lo scrittore. Inoltre: "Ci sarebbe ancora molto da dire sulla necessità e l'urgenza, nel nostro paese, di un lavoro culturale portato avanti con perseveranza. Io credo che il grido «la patria è in pericolo!» sia meno sconvolgente di questo altro grido: «Cittadini! La cultura è in pericolo! $\gg^{14}$

La concezione romantica della rivoluzione culturale si scontra con la rozza realtà dei primi eventi rivoluzionari, suscitando in $\mathrm{M}$. Gor'kij le prime delusioni, le preoccupazioni e i timori; sentimenti che si traducono in appassionati discorsi, richiami, avvertimenti, domande.

"Dove è possibile vedere messa in pratica la «nuova» morale, la morale del socialismo?" si interroga l'autore. "È indubbio che i miei rimproveri mi attireranno la sprezzante risposta di uno di questi signori della «Realpolitik»: «Ma cosa pretende? È questa, la rivoluzione sociale! ».

No, io non vedo affatto, in questa esplosione di istinti zoologici, elementi chiaramente espressi dalla rivoluzione sociale. È una rivolta russa senza veri socialisti; la psicologia socialista non vi ha parte alcuna". ${ }^{15}$

E proprio in questo momento viene rivisto il ruolo dell'intelligencija e vengono rivalutate le sue potenzialità: proprio l'intelligencija, dichiara, deve assumersi il gravoso compito della cura spirituale del popolo. A differenza di V. Lenin che disprezzava l'intelligencija definendola lacché del capitalismo, Gor'kij parlava con entusiasmo e fiducia delle forze intellettuali della Russia che svolgevano un'attività intensa, considerandola il pegno e l'inizio della rinascita spirituale del paese. ${ }^{16}$

Tra tanti argomenti affrontati negli articoli, un interesse particolare presentano quelli dove M. Gor'kij polemizza con i bolscevichi, di-

14. Ibidem. pp. 64-65.

15. Maksim Gor'kij. Pensieri intempestivi, 1917-1918, Ed. Jaca Book, Milano, 1978, p. 98.

16. Neizvestnyj Gor'kij, M., Nasledie, 1994, pp. 29-33. 
chiarando spesso il proprio profondo dissenso e la propria opposizione alla linea, definita folle, dei commissari del popolo.

Prendendo in esame l'impegno dei rivoluzionari, li distingue in due tipi: il rivoluzionario eterno e il rivoluzionario temporaneo, quello del momento contingente.

"Il primo, che incarna il principio rivoluzionario di Prometeo, appare come l'erede spirituale di tutta la massa di idee che ha condotto l'umanità alla perfezione, e tali idee sono impresse non solo nel suo spirito, ma anche nei suoi sentimenti, persino nel dominio del subcosciente. (...)"

"Il rivoluzionario temporaneo, quello dell'istante presente, è un uomo che sente con una sensibilità morbosa le offese e le ingiustizie sociali, le sofferenze inflitte dagli uomini. (...) offre lo spettacolo triste e talvolta tragicomico di una creatura venuta tra gli uomini, sembrerebbe, per deformare, denigrare, abbassare fino al ridicolo, al volgare e all'assurdo il contenuto culturale, umanitario ed universale delle idee rivoluzionarie.(...) È completamente impregnato, come una spugna, del sentimento di vendetta, e vuole restituire centuplicate le offese. Le idee che ha accettato solo mentalmente, che non si sono radicate nel suo animo, si trovano in contraddizione diretta ed insolubile con i suoi atti; (...) Si comporta con la gente come uno scienziato meschino coi cani o colle rane destinati ai suoi crudeli esperimenti scientifici, con questa differenza però, che per quanto miserabile, uno scienziato fa soffrire inutilmente degli animali nell'interesse dell'uomo, mentre un rivoluzionario temporaneo è ben lontano dall'essere sempre sincero nei suoi esperimenti compiuti sull'uomo. Gli uomini sono per lui un materiale tanto più utilizzabile quanto meno è spiritualizzato." ${ }^{\prime 17}$ Così vede lo scrittore numerosi rivoluzionari che distruggono, rovinano, rubano, compiono crimini e non ha paura di dichiararlo apertamente.

L'autore accusa i bolscevichi definendo amorale il loro rapporto con il popolo considerato l'oggetto d'un esperimento disumano.

Ai rimproveri di parlar male del popolo a cui appartiene risponde fermamente: "Io ho il diritto di dire la verità amara e scottante sul popolo, e sono persuaso che sia assai meglio per lui che questa verità la dica io per primo, piuttosto che i suoi nemici (...) Non bisogna credere che il popolo sia sano e giusto soltanto perché è martire; (...) E non bisogna rifiutarsi

17. Maksim Gor'kij, Pensieri intempestivi, 1917-1918, Ed. Jaca Book, Milano, 1978, pp. 260-262. 
di constatare che oggi il «popolo» ha acquistato il diritto di esercitare la violenza fisica sugli uomini, ed è divenuto un torturatore altrettanto crudele e feroce dei suoi antichi carnefici." 18

Molto presto Gor'kij si rende conto che l'unico modo che rimane ai bolscevichi per mantenere il potere è la dittatura con tutte le conseguenze che porta. $\mathrm{E}$ anche in questo caso la coraggiosa voce dello scrittore lancia le accuse: "Lo sterminio in massa di tutti gli oppositori è un vecchio e sperimentato sistema della politica interna dei governi russi. Da Ivan il Terribile a Nicola II, tutti i nostri capi politici hanno usato liberamente e largamente questo mezzo pratico e semplice per combattere la sedizione perché Vladimir Lenin dovrebbe rinunciare ad un metodo tanto semplificatore? Non soltanto non vi rinuncia, ma annuncia assai francamente che non indietreggerà di fronte a nulla per sterminare i nemici." ${ }^{19}$

Come inaspettate ed assolute novità si leggono in questi articoli e nei documenti successivamente resi pubblici, ${ }^{20}$ numerosi giudizi molto forti sui capi dei bolscevichi: Lenin, schiavo del dogma, viene accusato di fare, col popolo russo, un esperimento crudele, votato in anticipo all'insuccesso.

Già in una lettera del 1909 inviata a Lenin da Capri, insieme con una dichiarazione di rispetto e di stima, Gor'kij evidenzia un tratto caratteristico di Lenin scrivendo: “(...) per Lei ogni persona non vale più di un flauto con cui suonare la melodia preferita, Lei valuta ogni individuo dal punto di vista della sua utilità per Lei e per la realizzazione di Suoi scopi, idee, compiti..." 21

Prima ancora un altro contemporaneo ebbe modo di conoscere abbastanza da vicino V. Ul'janov di ritorno dall'esilio siberiano, nel periodo (1899-1900) quando nasceva a Pskov il giornale Iskra, primo organo di stampa social-democratico. Scrive nel suo libro: "Arrivato a Pskov Lenin entrò a far parte del nostro circolo marxista diventando subito una figura centrale grazie alla sua erudizione nelle questioni economiche, particolarmente nella loro interpretazione in chiave marxista... Lenin non apparteneva a quegli uomini che ti colpivano per la forza e l'originalità del pensiero. Il suo pensiero si trovava rinchiuso nella sagoma delle idee marxi-

18. Maksim Gor'kij. Pensieri intempestivi, 1917-1918, Ed. Jaca Book, Milano, 1978, pp. 179-180.

19. Ibidem. p.167.

20. A partire dagli anni 1990 inizia il processo di revisione del tema Gor'kij - Lenin.

21. Neizvestnyj Gor'kij, M., Nasledie, 1994, p. 25. 
ste. Impressionavano molto la sua memoria fenomenale e le capacità straordinarie... Non saprei dire se Lenin avesse una ampia istruzione. Era talmente assorbito dalle questioni sociali e politiche che non affrontava mai con noi altri argomenti. Non riesco ad immaginarlo mentre conversava di poesia, pittura, musica e meno ancora dell'amore, delle complesse emozioni umane o delle piccolezze di vita quotidiana non legate alla cospirazione. Gli era del tutto estraneo l'interesse per l'uomo. Parlando con lui sentivo che si interessava a me solo in quanto possibile compagno di idee da utilizzare per la lotta rivoluzionaria.(...) La mia personalità, i miei sentimenti ed emozioni non lo interessavano affatto. (...) La freddezza di Lenin nei confronti della gente si notava..."22

Gor'kij che conosceva bene Lenin, evidenzia che questa tragedia ineluttabile non turba affatto il leader bolscevico: "La vita nella sua complessità è estranea a quest'uomo: egli non conosce le masse popolari; non ha mai vissuto col popolo, ma ha imparato, sui libri, come muovere le masse, soprattutto come eccitare furiosamente gli istinti delle folle. La classe operaia è per Lenin ciò che il minerale è per l'operaio metallurgico. È possibile, date le circostanze, fabbricare con questo minerale uno stato socialista? Tutto lascia pensare di no; ma costui dice "perché non provare?" Cosa rischia Lenin se il tentativo fallisce?

Egli lavora come un chimico nel suo laboratorio, con la differenza che il chimico utilizza una materia morta ed ottiene col suo lavoro dei risultati preziosi per la vita, mentre Lenin lavora su di una materia viva, e conduce la rivoluzione alla morte". ${ }^{23}$

"Lenin, Trockij e i loro compagni sono già contaminati dal veleno del potere", spesso si incontrano avvertimenti: "La classe operaia non può non comprendere che Lenin sta semplicemente per tentare un esperimento sulla sua pelle e sul suo sangue", oppure "Lenin non è un mago dagli innumerevoli poteri, ma un prestigiatore dalla mente fredda che non tiene in considerazione né la vita né l'onore del proletariato". ${ }^{24}$

Sono davvero numerosi gli avvertimenti, le indicazioni nelle descrizioni della drammatica situazione che si era creata in Russia nel 1917, delle scelte da compiere e delle responsabilità di chi si era messo alla

22. V.A. Obolenskij. La mia vita. I miei contemporanei. (Moja žizn'. Moi sovremenniki), YMCA-PRESS, Paris, 1988, pp. 177-178.

23. Maksim Gor'kij. Pensieri intempestivi, 1917-1918, Ed. Jaca Book, Milano, 1978 , p. 125.

24. Ibidem., pp. 114-115. 
guida della nazione nel momento di svolta. Si capisce inoltre il motivo per cui "le note sulla cultura" restarono chiuse negli archivi come un materiale pericoloso. Non è difficile immaginare come possano essere percepite oggi queste ed altre affermazioni di Gor'kij disseminate nei Pensieri intempestivi alla luce della esperienza storica del XX secolo.

Bisogna tener presente che nei Pensieri intempestivi Gor'kij è stato l'interprete autentico sia dell'intelligenzia russa socialista e liberale che di una élite illuminata della classe operaia. Nelle pubblicazioni del 1917 1919 non troveremo quella presuntuosa convinzione ed univocità che caratterizzavano gli articoli del periodo precedente - Zametki o meščanstve (Note sulla piccola borghesia) (1905), Razrušenie ličnosti (Distruzione della personalità) (1909) - e quelli degli anni 1930 (creati nella "prigionia di Stalin"). ${ }^{25}$

La sostanza di questa pubblicistica sta nell'affrontare le più scottanti, difficili e spesso irrisolvibili questioni della nostra esistenza, nella ricerca di risposte.

I Pensieri inopportuni sono stati, purtroppo, profetici, e non hanno perso il loro significato, rimangono una specie di monumento pubblicistico al suo tempo.

La rilettura odierna del testo gor'kiano ci permette non solo di verificare la vera posizione di M. Gor'kij nei confronti degli eventi rivoluzionari, ma ci ripropone questioni che rimangono attuali e di vitale importanza anche per le società contemporanee.

Continua nel frattempo lo studio dell'eredità gor'kiana e della sua contraddittoria personalità. ${ }^{26}$

Recentemente è uscita una nuova biografia di M. Gor'kij. ${ }^{27}$ In una intervista l'autore lo definisce senza ombra di dubbio la più importante figura a cavallo di due secoli: "In teoria è possibile immaginare quest'epoca senza chiunque, persino senza A. Blok. Certo, si sentirebbe un enorme vuoto, $\mathrm{ma}$ in generale l'epoca si conserverebbe, senza Gor'kij no. E' quel punto che tiene insieme tutti i fili di forza dell'epoca. Prova a togliere questo punto - tutto crollerà”.

25. A partire dalla fine degli anni '20, Gor'kij comincia a riconoscere pubblicamente il carattere erroneo della sua opinione d'una volta secondo cui i bolscevichi e Lenin avrebbero portato alla rovina la classe operaia e l'intelligencija socialista, gettandole nel caos dell'anarchia.

26. L. Spiridonova. M. Gor'kij: novyi vzgljad (M. Gor'kij: una nuova visione), M., IMLI RAN, 2004.

27. Basinskij P. Gor'kij, M., Molodaja Gvardija, 2006. 